\title{
LETTER
}

\section{Clinical features and short-term outcomes of 18 patients with corona virus disease 2019 in intensive care unit}

\author{
Jianlei Cao ${ }^{1}$, Xiaorong Hu${ }^{1}$, Wenlin Cheng ${ }^{1}$, Lei Yu ${ }^{2}$, Wen-Jun Tư ${ }^{3 *}$ and Qiang Liư ${ }^{3^{*}}$
}

(C) 2020 Springer-Verlag GmbH Germany, part of Springer Nature, corrected publication 2020

\section{Dear Editor,}

In December, 2019, a series of pneumonia cases of unknown cause emerged in Wuhan, Hubei, China [1] and further a novel coronavirus was isolated from lower respiratory tract samples, which was further named Severe Acute Respiratory Syndrome Coronavirus 2 (SARSCoV-2) [2]. The SARS-CoV-2 disease was officially given the name of Corona Virus Disease 2019 (COVID-19) on 11 February, 2020. In this study, we examine which treatments are being used, assess clinical and laboratory features and short-term outcomes of patients with COVID-19 in intensive care unit (ICU).

This retrospective case series involved 102 adult patients with COVID-19 admitted to Wuhan University Zhongnan Hospital in Wuhan, China, between January 3 and February 1, 2020. All those patients received a laboratory-confirmed SARS-CoV-2 infection diagnosis [3]. Epidemiological, clinical, radiological characteristics, underlying diseases, laboratory tests at admission and during hospitalization, treatment, complications and outcomes data were collected [3, 4]. Patients outcomes (discharge or death) and admission to ICU (yes or no) were followed up at discharge until February 15, 2020. The study was approved by Zhongnan Hospital Ethics Committee, and oral consent was obtained from patients or relatives.

Demographic details of the included 102 patients are shown in the supplementary table 1 . All patients were

\footnotetext{
*Correspondence: tuwenjun@irm-cams.ac.cn; liuqiang@irm-cams.ac.cn

${ }^{3}$ Institute of Radiation Medicine, China Academy of Medical Science

and Peking Union Medical College, No. 238, Baiti Road, Tianjin 300192, People's Republic of China

Full author information is available at the end of the article

Jianlei Cao and Xiaorong Hu have contributed equally to this work.
}

isolated. Seventeen patients died (discharge mortality, 16.7\%; 95\% confidence interval [CI], 9.4-23.9\%), and eighteen patients were admitted to the ICU with a rate of $17.6 \%$ (95\% CI, 10.2-25.0\%). The reasons for admission included need for mechanical ventilation $(N=6)$, breathing rate increases/oxygen saturation $<90 \% /$ no-compliance with noninvasive ventilator $(N=7)$, and combined shock and/or organ failure $(N=5)$. The timeline of SARSCoV-2 onset in ICU patients is shown in Fig. 1. The ICU patients were older (66[54-76] vs. 31[35-62]) and more likely exposed to source of transmission $(66.7 \%$ vs. $40.2 \%)$ when compared to the non-ICU patients. Health care workers were less likely admitted to the ICU ( $0 \%$ vs. 28.2\%). ICU patients more likely suffered from comorbidities (Any [100.0\% vs. 34.5\%]; hypertension [55.5\% vs. $21.4 \%]$; and chronic liver disease [11.1\% vs. $0 \%]$ ). Those patents needed more time to confirm the laboratory diagnosis (12[7-19] vs. 8[4-11]). They also needed longer hospital stays $(15[8-21]$ vs. $10[7-14])$ and more medical expenses $(62,556[48,938-160,629]$ vs. $12,808[8166-$ 27,691] CNY, See the supplementary table 1).

As shown in the supplementary table 1, the treatment in ICU included the administration of a antiviral therapy $(100.0 \%)$, the use of antibiotics (100.0\%), glucocorticoid therapy (61.1\%), oxygen inhalation (61.1\%), noninvasive ventilation $(11.1 \%)$, invasive mechanical ventilation (38.9\%), extracorporeal membrane oxygenation (16.7\%) and CRRT (22.2\%). Furthermore, there were no significant difference in drugs treatment, oxygen inhalation and noninvasive ventilation between ICU group and non-ICU group $(P>0.05$ all). ICU patients received more intensive treatment with invasive mechanical ventilation $(38.9 \%$ vs. $4.8 \%)$, extracorporeal membrane oxygenation $(16.7 \%$ vs. $0 \%)$ and CRRT (22.2\% vs. $2.4 \%)$. They also more likely

\section{Springer}


a

Discharge

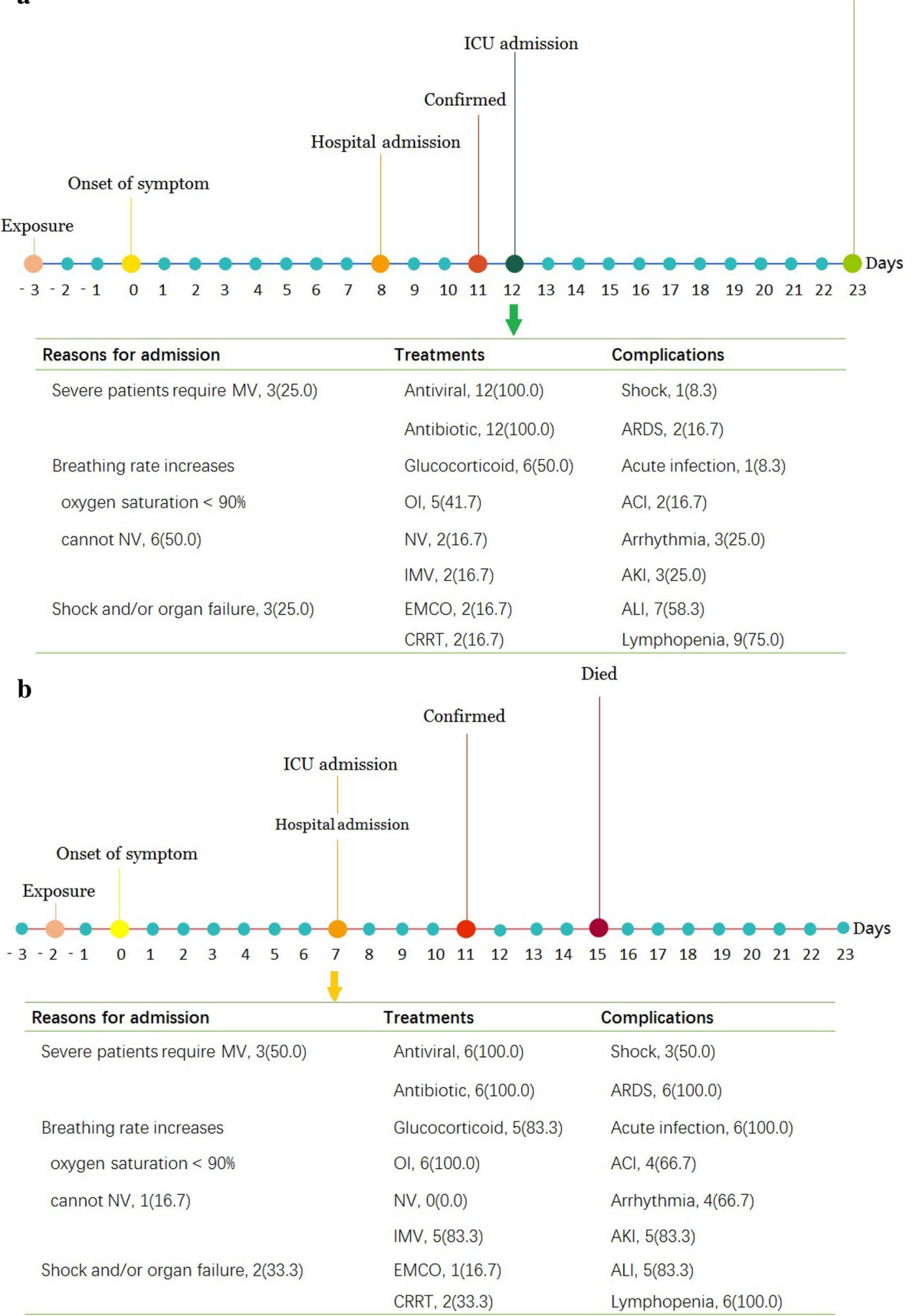


(See figure on previous page.)

Fig. 1 The timeline of SARS-CoV-2 onset in ICU patients. a Timeline of SARS-CoV-2 onset in ICU survivors $(N=12)$. b Timeline of SARS-CoV-2 onset in ICU non-survivors $(N=6)$. The results were presented as number $(\%)$. The onset of symptom was defined as day 0 . The points represent the median value. ICU, intensive care unit; SARS-CoV-2, severe acute respiratory syndrome coronavirus 2; MV, mechanical ventilation; NV, noninvasive ventilation; IMV, invasive mechanical ventilation; EMCO, extracorporeal membrane oxygenation; Ol, oxygen inhalation; ACl, acute cardiac injury; AKI, acute kidney injury; ALI, acute liver injury

suffered from in-hospital complications (ARDS [44.4\% vs. $14.3 \%]$; acute infection [38.9\% vs. $11.9 \%$; acute cardiac injury [33.3\% vs. $10.7 \%]$; arrhythmia [38.9\% vs. $13.1 \%]$; acute kidney injury [44.4\% vs. $14.3 \%]$ and acute liver injury [66.7\% vs. $26.2 \%])$. ICU patients had a higher mortality rate than non-ICU patients $(33.3 \%$ vs. $13.1 \%)$, but this difference was not significant $(P=0.081)$.

Our results suggest that ICU patients suffer at admission from more comorbidities and develop many complications due to hospitalization. During hospitalization they receive more aggressive treatment, and can result in a similar mortality when compared to non-ICU patients. We found that $17.6 \%$ of patients required admission to the ICU and $16.7 \%$ died. A previous study including 138 patients with COVID-19 showed that $26 \%$ of patients required admission to the ICU and $4.3 \%$ died [3]. Another study reported that $23 \%$ of patients with COVID-19 required admission to the ICU and 11.0\% died [4]. It should be noted that most patients in those two studies were still hospitalized at the time of manuscript submission [3, 4]. Our hospital is one of the major tertiary teaching hospitals and is responsible for the treatment of critically ill patients with COVID-19. Thus, our cohort might represent the more severe COVID-19 patients and the rates of death and ICU admission may be overestimated. A recent large-sample and multicenter study showed that only $5 \%$ of the included COVID- 19 patients were admitted to ICU and $1.36 \%$ succumbed [5].

\section{Electronic supplementary material}

The online version of this article (https://doi.org/10.1007/s00134-020-05987-7) contains supplementary material, which is available to authorized users.

\section{Author details \\ ${ }^{1}$ Department of Cardiology, Zhongnan Hospital, Wuhan University, Wuhan, People's Republic of China. ${ }^{2}$ Department of Infectious Diseases, The Fourth Affiliated Hospital of Harbin Medical University, Harbin, People's Republic of China. ${ }^{3}$ Institute of Radiation Medicine, China Academy of Medical Science and Peking Union Medical College, No. 238, Baiti Road, Tianjin 300192, People's Republic of China.}

\section{Acknowledgements}

We thank all patients included in this study. We are really grateful to all the health workers around the world. Their expertise \& humanity are fundamental to stop SARS-COV-2 from spreading further. .

\section{Author contributions}

JC and XH contributed equally as the co-author. WJT and QL contributed equally as senior authors. JC and WJT had full access to all of the data in the study and take responsibility for the integrity of the data and the accuracy of the data analysis. JC, XH, WC, LY, WJT, and QL were involved in concept and design. JC, XH, WC, LY, WJT, and QL contributed to acquisition, analysis, or interpretation of data. JC, XH, WJT, and QL were involved in drafting of the manuscript. JC and $L Y$ were involved in critical revision of the manuscript for important intellectual content. JC and WJT contributed to statistical analysis. $J C, X H, W C, W J T$, and QL were involved in administrative, technical, or material support. JC, XH, and WC contributed to supervision. WJT and QL obtained funding.

\section{Funding}

This study was supported by funding from CAMS Innovation Fund for Medical Science (Dr, Liu Q, No. 2017-I2M-1-016; Dr. Tu, 2019-12M-2-006); Natural Science Foundation of Tianjin (Dr. Tu, No. 19JCYBJC26600) and China Postdoctoral Science Foundation funded project (Dr. Tu, No. 2019M660921).

\section{Data availability}

Data available can be obtained from the corresponding author.

\section{Compliance with ethical standards}

\section{Conflicts of interest}

None reported.

\section{Role of the funders/sponsors}

The study funders/sponsors had no role in the design and conduct of the study; collection, management, analysis, and interpretation of the data; preparation, review, or approval of the manuscript; and decision to submit the manuscript for publication.

\section{Consent for publication}

Not applicable.

\section{Publisher's Note}

Springer Nature remains neutral with regard to jurisdictional claims in published maps and institutional affiliations.

Accepted: 21 February 2020

Published online: 2 March 2020

\section{References}

1. Huang C, Wang Y, Li X, Ren L, Zhao J, Hu Y, Zhang L, Fan G, Xu J, Gu X, Cheng Z (2020) Clinical features of patients infected with 2019 novel coronavirus in Wuhan, China. Lancet 395:497-506

2. Zhu N, Zhang D, Wang W, Li X, Yang B, Song J, Zhao X, Huang B, Shi W, Lu R, Niu P (2020) A novel coronavirus from patients with pneumonia in China, 2019. N Engl J Med. https://doi.org/10.1056/NEJMoa2001017

3. Wang D, Hu B, Hu C, Zhu F, Liu X, Zhang J, Wang B, Xiang H, Cheng Z, Xiong Y, Zhao Y (2020) Clinical characteristics of 138 hospitalized patients with 2019 novel coronavirus-infected pneumonia in Wuhan, China. JAMA. https://doi.org/10.1001/jama.2020.1585

4. Chen N, Zhou M, Dong X, Qu J, Gong F, Han Y, Qiu Y, Wang J, Liu Y, Wei Y, Yu T (2020) Epidemiological and clinical characteristics of 99 cases of 2019 novel coronavirus pneumonia in Wuhan, China: a descriptive study. Lancet 395:507-513

5. Guan WJ, Ni ZY, Hu Y, Liang WH, Ou CQ, He JX, Liu L, Shan H, Lei CL, Hui DS, Du B (2020) Clinical characteristics of 2019 novel coronavirus infection in China. medRxiv. https://doi.org/10.1101/2020.02.06.20020974 